\title{
Packing-limited growth of irregular objects
}

\author{
Peter Sheridan Dodds ${ }^{1}$ 周 and Joshua S. Weitz ${ }^{2,3}$, 周 \\ ${ }^{1}$ Institute for Social and Economic Research and Policy, Columbia University, New York, NY 10027. \\ 2 Department of Earth, Atmospheric and Planetary Sciences, \\ Massachusetts Institute of Technology, Cambridge, MA 02139. \\ 3 Department of Physics, Massachusetts Institute of Technology, Cambridge, MA 02139.
}

(Dated: November 18, 2018)

\begin{abstract}
We study growth limited by packing for irregular objects in two dimensions. We generate packings by seeding objects randomly in time and space and allowing each object to grow until it collides with another object. The objects we consider allow us to investigate the separate effects of anisotropy and non-unit aspect ratio. By means of a connection to the decay of pore-space volume, we measure power law exponents for the object size distribution. We carry out a scaling analysis, showing that it provides an upper bound for the size distribution exponent. We find that while the details of the growth mechanism are irrelevant, the exponent is strongly shape dependent. Potential applications lie in ecological and biological environments where sessile organisms compete for limited space as they grow.

PACS numbers: 02.70.Rr, 05.10.-a, 87.23.Cc, 87.23.-n, 81.10.Aj
\end{abstract}

\section{INTRODUCTION}

In a previous work [1], we examined packings formed by spheres growing in $d$ dimensions, immediately stopping upon contact with another sphere. We termed this to be packing-limited growth (PLG). Here, we address the question of what happens when we consider irregular (i.e., non-spherical) objects of similar shape growing in $d=2$ dimensions. We focus in particular on collisions of growing rectangles. By combining rectangles, we are able to form and examine a range of shapes whose growth patterns vary broadly in radial anisotropy. Whereas in [1] we found universality classes depending only on dimension $d$, we find here that marked non-universal behavior arises when shapes are varied.

Our problem finds several motivations. First, this is a relatively unexplored kind of packing. Packings are generally static or randomly generated [2]: physical mechanisms are only seldomly connected with the creation of

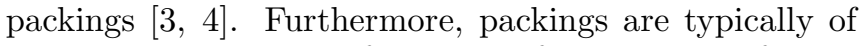
monodisperse objects of the same form or taken from a small set of forms [5, 6. From a physical point of view, two-dimensional packings of growing objects may be of use in modeling or understanding certain biological and ecological patterns. We consider the geometric approach presented here as a preliminary step towards describing how shape alters the size distributions of populations.

In section III we describe the various objects we construct from rectangles. Although not all of these objects have obvious physical parallels, they present a range of typological cases from which direct applications and comparisons may be sought. In section III, we provide a scal-

*Electronic address: peter.dodds@columbia.edu

${ }^{\dagger}$ Electronic address: jsweitz@segovia.mit.edu; (Please direct correspondence to both authors.) ing analysis that empirically appears to be exact for $d \geq 4$ in the case of hyperspheres [1] (details of the calculations for this section are given in Appendix A). We report the results of our numerical investigations in section IV along with a discussion of the failings of the scaling theory. We conclude the paper in section $\square$ and outline our algorithm for packing rectangles in Appendix B.

\section{IRREGULAR OBJECTS CONSIDERED}

The building block shape of the objects we consider is the rectangle. We denote the aspect ratio as $a$, defining all rectangles such that $a \geq 1$. We record rectangle size $r$ as half the length of the long side, the dimensions then being $2 r$ and $2 r / a$. Examples of each object are provided in the packings of Figures 11 and 2.

For $a=1$, we have squares and as $a \rightarrow \infty$, rectangles effectively become line segments and the packing becomes one of fitting $d=1$ objects into a $d=2$ volume. Rectangles afford a basic example of anisotropic growth since the long side grows at a rate slower than the short side by a factor of $1 / a$. The growing square is distinguished from the rectangle since the former expands uniformly perpendicular to its edges. However, in comparison to disks, the growth rate of edge points relative to the center of both squares and rectangles is non-uniform.

The simplest combination of two rectangles is a cross (Figures 2 $\mathrm{A}$ and $2 \mathrm{~B}$ ) and a natural generalization is the $2 n$-spoke object (with $n=1$ being a rectangle and $n=2$ being a cross, see Figure 2 $\mathrm{C}$ ). Each $2 n$-spoke object is then part of a family of shapes indexed by $a$.

The last object we consider is an eight-pointed star as shown in Figure 2D. This object is formed by two squares overlaid at angle of $\pi / 4$ to each other.

All objects are packed using the approach of Manna [7]: objects are added sequentially and allowed to instantly grow so as to just reach the existing packing structure. 

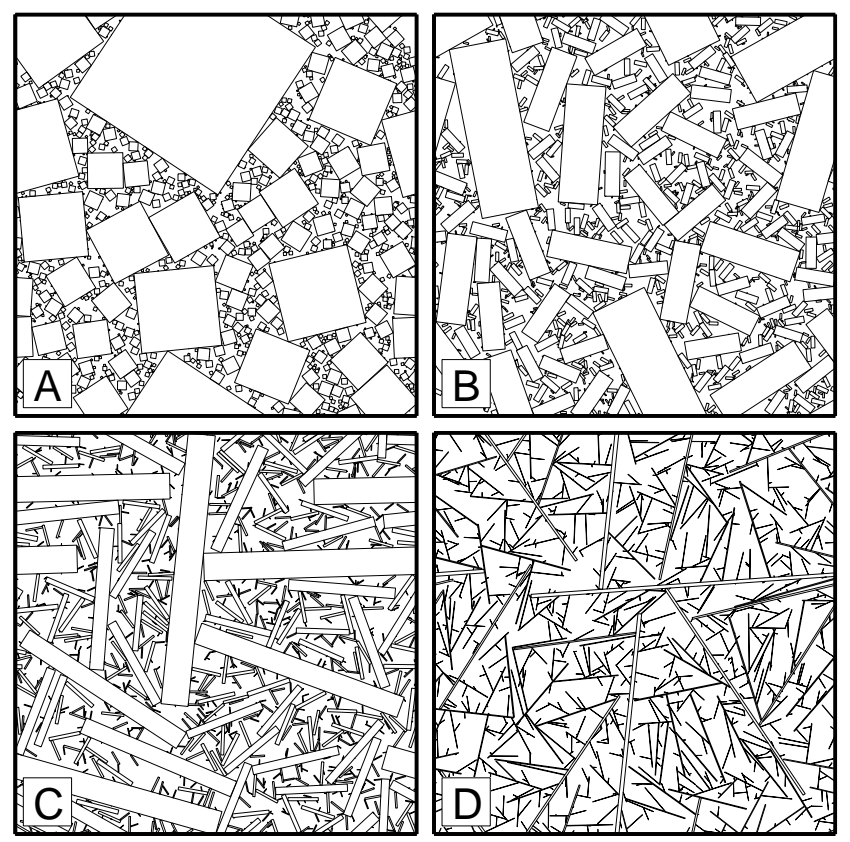

FIG. 1: Rectangle packings of the unit square for varying aspect ratio $a$ created using the packing-limited growth mechanism. Each packing consists of 1000 rectangles with periodic boundary conditions being imposed. The aspect ratios corresponding to the packings are (A) $a=1$, (B) $a=3$, (C) $a=10$, and (D) $a=100$. The first three packings are initialized with four randomly placed and oriented rectangles with longest side length 0.25 , with eight such rectangles being used in the fourth packing.

We have observed and argued [1] that the value of the size distribution exponent and all other related exponents is independent of the growth dynamic.

In the context of packing, spheres make for straightforward calculations since the contact point between any two colliding spheres occurs along the line through their centers. Rectangles constitute a relatively simple generalization of spheres from a numerical point of view, hence our use of them here.

\section{SCALING THEORY}

In [1], we argued that a simple scaling assumption may be made regarding the form of $P(r ; n)$, the distribution of sphere sizes after $n$ objects have been packed. We take $P(r ; n)$ to be described by a power law for radii above a cutoff value $r_{c}$ and uniform below:

$$
P(r ; n)= \begin{cases}\frac{\alpha-1}{\alpha} r_{c}^{-1} & \text { for } r<r_{c} \\ \frac{\alpha-1}{\alpha} r_{c}^{-1}\left(\frac{r}{r_{c}}\right)^{-\alpha} & \text { for } r \geq r_{c} .\end{cases}
$$

The tail of the distribution is fixed and the distribution fills in $\left(r_{c}\right.$ decreases with $\left.n\right)$ with the $(n+1)$ th object being chosen from within the pore space. The size distribution $P(r ; n)$ is connected to the probability of inserting
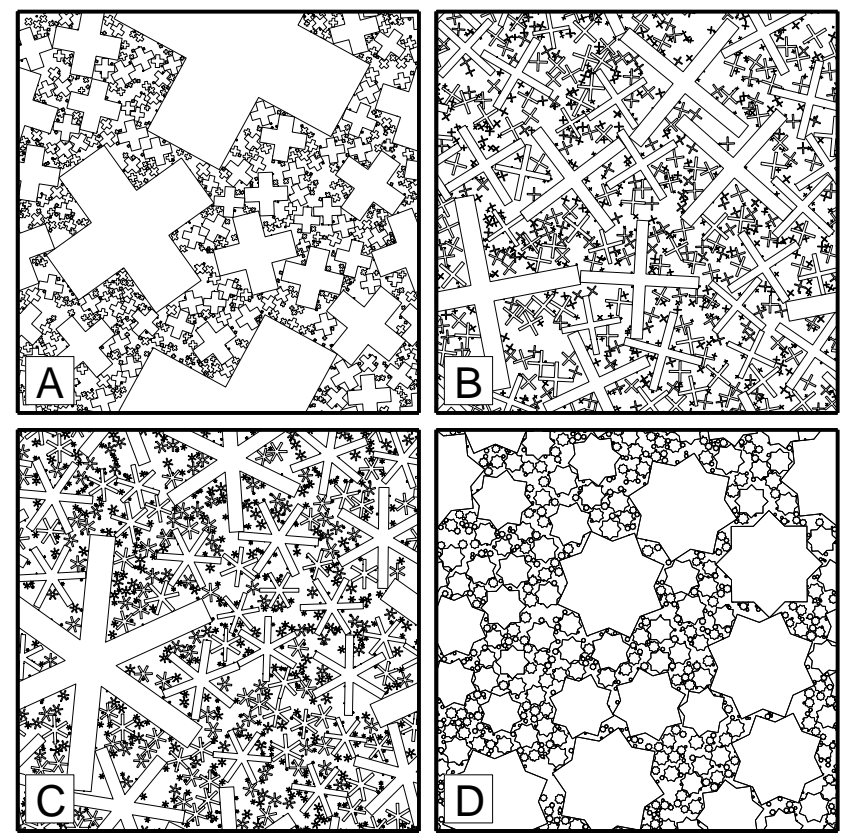

FIG. 2: Packings of irregular objects formed by combinations of rectangles. As per Figure 1, each packing contains 1000 objects and the boundary conditions are periodic. In (A) and (B), the crosses are formed by two rectangles at right angles with aspect ratios $a=3$ and $a=10$ respectively. Plot (C) shows a packing of six-legged stars composed of three rectangles with aspect ratio $a=10$. In (D), the packing object is an eight-pointed star formed by two overlapping squares set at an angle of $\pi / 4$ to each other.

an object of size $r$ after $n$ objects have been deposited, $P_{\text {ins }}(r ; n)$. Since the probability of adding a sphere of vanishing radii must be proportional to the total surface area of the existing spheres $S(n)$, we were able to estimate $P_{\text {ins }}(r ; n)$ as [1]

$$
P_{\text {ins }}(r ; n)=\frac{S(n)}{\Phi(n)}, \quad 0 \leq r \leq r_{c},
$$

where $\Phi(n)$ is the pore space volume. Using equation (11) to calculate $S(n)$ and $\Phi(n)$ and requiring that $P_{\text {ins }}(r ; n)$ be normalized, we obtained the estimate $\alpha=11 / 4$ for disks.

More generally in $d=2$ dimensions, if we now write area as $A=\rho r^{2}$ and perimeter as $L=\sigma r$, we find the scaling exponent to be

$$
\hat{\alpha}=\frac{9 \sigma+4 \rho}{3 \sigma+2 \rho} .
$$

Setting $\sigma=2 \pi$ and $\rho=\pi$, we recover $\alpha=11 / 4$ for disks. Note that the probability of adding a non-spherical object is still proportional to $S(n)$ in the limit of $r \rightarrow$ 0 , regardless of the object's shape. For example, it is irrelevant that an added square of side length $2 r$ may be oriented such that its center is between $r$ and $\sqrt{2} r$ away 


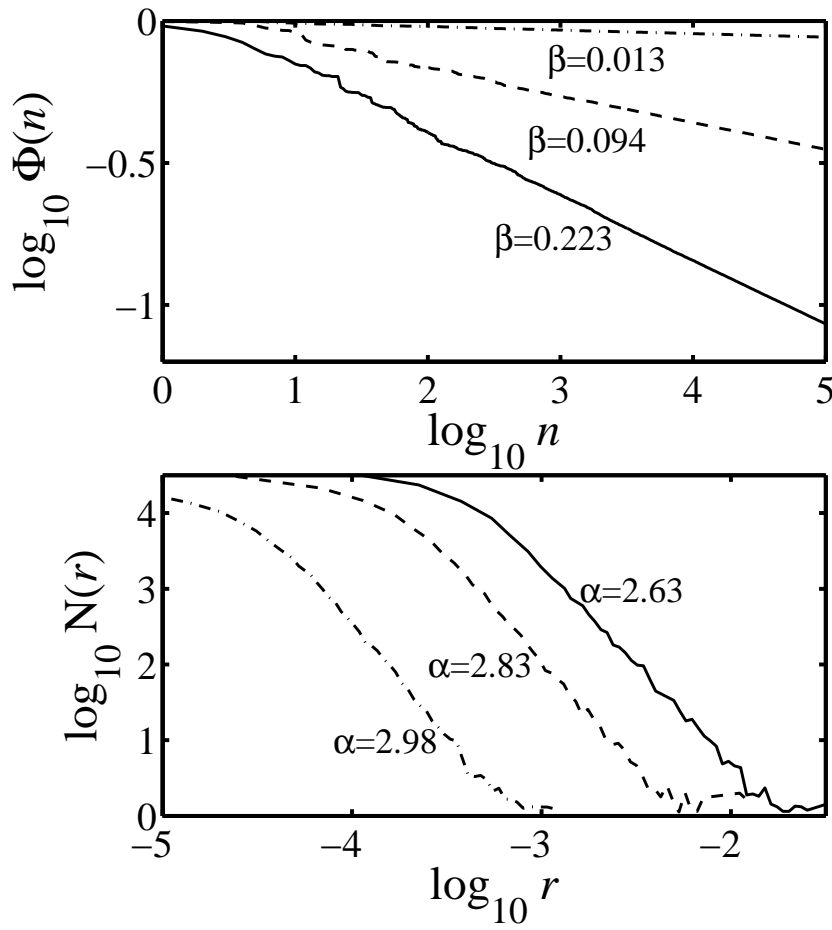

FIG. 3: For rectangle packings of $10^{5}$ objects, plots of $\Phi(n)$, the decay of pore space as a function of number of rectangles added $n$, and $N(r)$, the frequency of objects of size $r$, for rectangles. In both plots, the rectangles have aspect ratios $a=1$ (solid line), $a=10$ (dashed line) and $a=100$ (dot-dash line). $N(r)$ is binned in log-space for clarity. The exponent $\alpha$ (in $N(r) \propto r^{-\alpha}$ ) increases with aspect ratio $a$, limiting to 3 as $a \rightarrow \infty$. Correspondingly, $\beta$ decreases towards 0 (see equation (4))

from its point of contact with the existing packing. Also note that as $a \rightarrow \infty$, the theoretical prediction tends to $\hat{\alpha}=3$, in agreement with a previous determination of an upper bound for polydisperse packings [8]. Details of the calculations finding $\hat{\alpha}$ for the objects we consider here are to be found in Appendix A.

All other exponents depend on $\alpha$ via simple scaling relations [1]. In particular, the pore volume $\Phi(n)$ decays as $n^{-\beta}$ with the connection between $\beta$ and $\alpha$ being

$$
\alpha=1+\frac{2}{1+\beta} .
$$

We use this equation to calculate theoretical estimates of $\beta$, denoting them by $\hat{\beta}$. Since direct measurement of $\beta$ is a significantly more robust exercise than determining $\alpha$ from $P(r)$, we employ equation (㺼) in estimating $\alpha$ in the following section.

\section{NUMERICAL RESULTS}

For each shape, we generate statistics for single packings with $10^{5}$ objects. Some example distributions taken

\begin{tabular}{lcccc}
\hline \hline object & $\beta$ & $\hat{\beta}$ & $\alpha$ & $\hat{\alpha}$ \\
\hline disk & $0.278(1)$ & 0.1429 & $2.564(1)$ & 2.750 \\
square & $0.223(2)$ & 0.1429 & $2.635(2)$ & 2.750 \\
rectangle $(a=2)$ & $0.207(2)$ & 0.1000 & $2.656(2)$ & 2.818 \\
rectangle $(a=5)$ & $0.145(2)$ & 0.0526 & $2.746(2)$ & 2.900 \\
rectangle $(a=10)$ & $0.094(1)$ & 0.0294 & $2.828(2)$ & 2.943 \\
rectangle $(a=20)$ & $0.055(1)$ & 0.0156 & $2.897(2)$ & 2.969 \\
rectangle $(a=50)$ & $0.0242(3)$ & 0.0065 & $2.953(1)$ & 2.987 \\
rectangle $(a=100)$ & $0.0125(1)$ & 0.0033 & $2.975(1)$ & 2.993 \\
cross $(a=3)$ & $0.169(3)$ & 0.0847 & $2.710(3)$ & 2.844 \\
cross $(a=10)$ & $0.074(1)$ & 0.0307 & $2.862(2)$ & 2.940 \\
cross $(a=100)$ & $0.009(1)$ & 0.0033 & $2.983(2)$ & 2.993 \\
six spoke $(a=10)$ & $0.078(2)$ & 0.0318 & $2.855(3)$ & 2.938 \\
eight-pointed star & $0.213(2)$ & 0.1429 & $2.648(4)$ & 2.750 \\
\hline \hline
\end{tabular}

TABLE I: Estimates of $\alpha$, the exponent of the number distribution, $P(r) \propto r^{-\alpha}$, for irregular objects undergoing packinglimited growth. The results for disks are included for comparison 11. All other objects are combinations of rectangles (see Figures 11 and 2) with $a$ being the aspect ratio. The exponent $\beta$ is determined from $\Phi(n)$ and $\alpha$ is subsequently obtained using equation (4). Full details of the method of measuring $\beta$ are given in the text. Each measurement is for a single packing containing $10^{5}$ objects. Measurement errors reflect variation in the approach to a limiting value of the exponents using the method of Section IV. The scaling theory estimates of $\hat{\beta}$ and $\hat{\alpha}$, which are lower and upper bounds respectively, are calculated using equations (3), (4), and those given in Appendix A.

from rectangle packings for $a=1,10$, and 100 are shown in Figure 3. The distribution $N(r)$ is the unnormalized frequency distribution corresponding to $P(r)$. In the case of plain rectangles, we see that as $a$ increases, both the observed $\alpha$ and the theoretical estimate $\hat{\alpha}$ increase while $\beta$ and $\hat{\beta}$ accordingly decrease. Furthermore, for all the shapes considered we find that $\alpha<\hat{\alpha}$ for all finite $a$.

We note that measuring power law exponents is not a trivial procedure and here we take some care to ensure the validity of our results. While simple regression is the basic tool of analysis, the presence of, for example, crossovers and finite size cutoffs can substantially degrade the level of precision attainable. The method of measuring exponents we use here is based on examining a smoothed version of the derivative of the distribution as viewed in double logarithmic space (similar approaches are to be found in [9, 10]). For example, for $\Phi(n)$, we perform regression analysis on $\log _{10} \Phi(n)$ versus $\log _{10} n$ over a sliding, variable width window of values of $\log _{10} n$. Writing the upper and lower limits of this window as $\log _{10} n_{1}$ and $\log _{10} n_{2}$, we have $w=\log _{10} n_{2} / n_{1}$ being the width. In general, depending on the number of orders of magnitude spanned by the data, we would preferably choose $w$ in the range $0.5 \leq w \leq 3$. Here, we fix $w=1$ and calculate the "local" exponent $\beta\left(n_{1}\right)$ for each window. We find in all cases that $\beta\left(n_{1}\right)$ tends towards a 


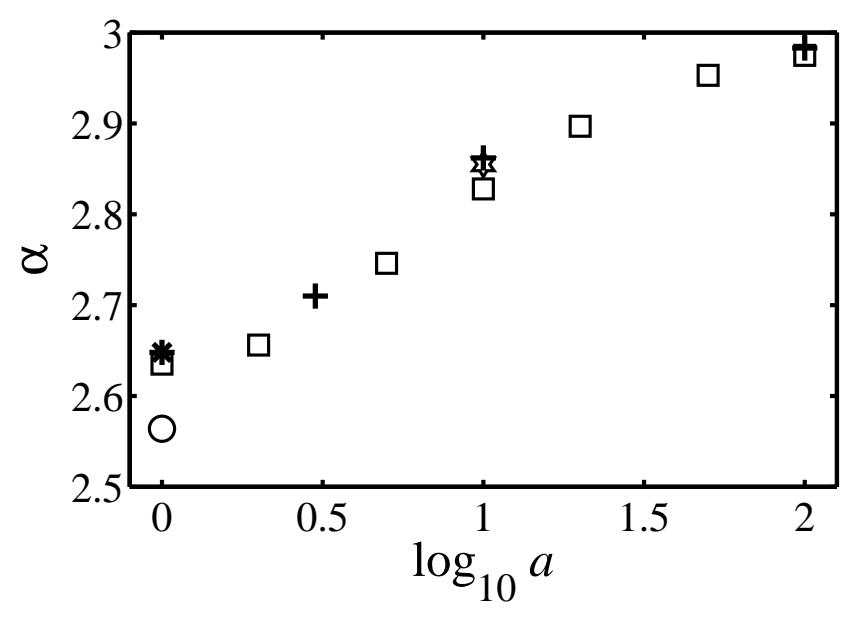

FIG. 4: Measured values of $\alpha$ as a function of aspect ratio $a$ and shape. The symbols correspond to disks (circles), rectangles (squares), crosses (plus sign), six-spoke (hexagon), and eight-pointed star (asterisk).

constant value. This indicates the scaling law is robust and further allows us to estimate $\beta$ along with an error based on the fluctuations observed for $\beta\left(n_{1}\right)$. Measured values of $\alpha$ and $\beta$ are recorded in Table 1 .

As we have noted above, our scaling theory approach appears to be exact for $d \geq 4$ dimensions in the case of hyperspheres and an overestimate of the true value of $\alpha$ for $d<4$. We therefore do not expect the scaling theory to be correct for non-spherical objects in $d=2$ dimensions. Indeed, in all cases, we observe the theoretical estimate $\hat{\alpha}$ is an overestimate of the measured $\alpha$. This direction of error makes sense in light of the scaling assumption made by equation (2). The actual form of $P_{\text {ins }}(r ; n)$ is not precisely uniform but rather rolls over less steeply than a step function around $r=r_{c}$. Given the manipulations that leads to (3), it can be argued that $\hat{\alpha} \geq \alpha$ must hold [1].

In Figure 1 , we show how $\alpha$ varies as a function of both aspect ratio $a$ of the constituent rectangles and the object's particular shape. The strongest influence is evidently aspect ratio with $\alpha$ varying from 2.564 (disks, $a=1$ ) up to 3 (rectangles, $n$-spokes, $a=\infty$ ). We observe a secondary effect due to the details of the shape. Square and the eight-pointed star packings have a value of $\alpha$ increased above that of disks. Cross packings have a higher value of $\alpha$ than do rectangles with the same aspect ratio.

\section{CONCLUDING REMARKS}

We have extended a model describing the interaction of growing disks [1] to the problem of growing irregular and anisotropic objects. In this model, the exponent characterizing the size distribution of objects is found to be independent of growth dynamics. However, the main result presented here is that the exponent is highly shape-dependent, adopting a continuous range of values $2.564 \leq \alpha \leq 3$. Ultimately, understanding how geometry impacts on the structure of plant communities will require imposing a notion of packing-limited growth onto a reasonable set of dynamics. The results here demonstrate that in so doing we must keep in mind that shape matters.

\section{Acknowledgments}

PSD acknowledges the support of the Columbia Earth Institute.

\section{APPENDIX A: SHAPE PARAMETERS FOR MEAN FIELD CALCULATIONS}

In this section, we derive the formulas for the area and perimeter of irregular objects in $d=2$. For general rectangles, we have area given by

$$
A_{\text {rect }}=4 r^{2} / a,
$$

and perimeter as

$$
L_{\text {rect }}=4(1+1 / a) r .
$$

Using equation (3), we therefore have the mean field estimate of $\alpha$ for rectangles as

$$
\hat{\alpha}_{\text {rect }}=3-\frac{2}{5+3 a}
$$

where writing the result in this fashion makes plain the limiting value of $\hat{\alpha}_{\text {rect }}=3$ for $a \rightarrow \infty$. For the $2 n$-spoke objects described above, area grows as

$$
A_{n \text {-spoke }}=2 n / a^{2}(2 a-\cot \pi / 2 n) r^{2},
$$

and perimeter is given by

$$
L_{n \text {-spoke }}=4 n / a(a+1-\cot \pi / 2 n) r,
$$

leading to the estimate

$$
\hat{\alpha}_{n \text {-spoke }}=\frac{13+9 a-(9+2 / a) \cot \pi / 2 n}{5+3 a-(3+1 / a) \cot \pi / 2 n} .
$$

Note that for $n=1$, we recover the result for rectangles in equation $\mathrm{A} 3$. Finally, for the eight-pointed star we have

$$
A_{8 \text {-point }}=2\left[2+(1-\tan \pi / 8)^{2}\right] r^{2},
$$

and

$$
L_{8 \text {-point }}=16(1-\tan \pi / 8) r,
$$

which yields

$$
\hat{\alpha}_{8-\text { point }}=\frac{42-40 \tan \pi / 8+2 \tan ^{2} \pi / 8}{15-14 \tan \pi / 8+\tan ^{2} \pi / 8}=11 / 8,
$$

the same as for disks and squares. 


\section{APPENDIX B: RECTANGLE COLLISIONS}

We describe an arbitrary rectangle in the $x-y$ plane as follows. The sides of the rectangle are $2 r$ and $2 r / a$ where $r>0$ is half the length of the long side of the rectangle and $a \geq 1$ is take the aspect ratio. The rectangle is centered at $\left(x_{0}, y_{0}\right)$ and rotated at an angle $\theta$. We take $\theta$ as the angle between the direction of the positive $x$-axis and the short axis of the rectangle (i.e., parallel to the side with length $2 r / a$ ) so that $0 \leq \theta<\pi$.

A rectangle described by $(r, a)$ and $\theta=0$ and $\left(x_{0}, y_{0}\right)=$ $(0,0)$ satisfies the equation

$$
\max \left|\left[\begin{array}{c}
a x \\
y
\end{array}\right]\right|=\max \left|\left[\begin{array}{ll}
a & 0 \\
0 & 1
\end{array}\right]\left[\begin{array}{l}
x \\
y
\end{array}\right]\right|=r .
$$

This is a remapping of a rectangle into a square of side length $2 r$. For an arbitrary rectangle, we can map it onto a basic square by recentering it at the origin, removing the rotation, and undoing the dilation by the aspect ratio $a$ :

$$
\max \left|\left[\begin{array}{ll}
a & 0 \\
0 & 1
\end{array}\right]\left[\begin{array}{cc}
\cos \theta & \sin \theta \\
-\sin \theta & \cos \theta
\end{array}\right]\left[\begin{array}{l}
x-x_{0} \\
y-y_{0}
\end{array}\right]\right|=r .
$$

Therefore, to determine whether or not an arbitrary point $(x, y)$ lies on or within a given rectangle, we need to check whether or not

$$
\max \left|\left[\begin{array}{ll}
a & 0 \\
0 & 1
\end{array}\right]\left[\begin{array}{cc}
\cos \theta & \sin \theta \\
-\sin \theta & \cos \theta
\end{array}\right]\left[\begin{array}{l}
x-x_{0} \\
y-y_{0}
\end{array}\right]\right| \leq r .
$$

In packing growing rectangles, the above is used to check if a newly seeded rectangle has been placed in pore space and not within an existing rectangle.

For a rectangle that passes this test, the next calculation is to determine how large it may grow preserving its aspect ratio and orientation so that it just reaches an existing rectangle.

To do this, we consider one arbitrary rectangle that does not cover the origin and find the size of largest rectangle centered at the origin with the short side along the $x$-axis such that the rectangles just touch. We will then generalize to any configuration by appropriate rotations.

First, the "growing" rectangle could hit the already existing one at any of the latter's corner points. The four corner points are given by

$$
\begin{gathered}
{\left[\begin{array}{l}
x_{0} \\
y_{0}
\end{array}\right]+r\left[\begin{array}{c} 
\pm 1 / a \cos \theta-\sin \theta \\
\pm 1 / a \sin \theta+\cos \theta
\end{array}\right],} \\
{\left[\begin{array}{l}
x_{0} \\
y_{0}
\end{array}\right]+r\left[\begin{array}{c}
\mp a \cos \theta+\sin \theta \\
\mp a \sin \theta-\cos \theta
\end{array}\right] .}
\end{gathered}
$$

The other possible collisions are between the corners of the added rectangle and the sides of the existing rectangle. There may be $0,1,2,3$ or 4 such interceptions ( 1 or 3 if the rectangles touch at corners). To calculate these points, we parametrize each side of the existing rectangle and find the interceptions with $y= \pm a x$. For an arbitrary line segment described by

$$
x=b_{1}+b_{2} t \quad \text { and } \quad y=c_{1}+c_{2} t,
$$

with $-1 \leq t \leq 1$, the intersection with $y= \pm a x$ occurs when

$$
t=\frac{-c_{1} \pm a b_{1}}{c_{2} \mp a b_{2}} .
$$

Parametrizing the sides of an arbitrary rectangle gives the following:

$$
\begin{gathered}
{\left[\begin{array}{l}
x \\
y
\end{array}\right]=\left[\begin{array}{l}
x_{0} \\
y_{0}
\end{array}\right]+r\left[\begin{array}{c}
-\sin \theta \\
\cos \theta
\end{array}\right]+\frac{r t}{a}\left[\begin{array}{c}
\cos \theta \\
\sin \theta
\end{array}\right],} \\
{\left[\begin{array}{l}
x \\
y
\end{array}\right]=\left[\begin{array}{l}
x_{0} \\
y_{0}
\end{array}\right]+r\left[\begin{array}{c}
\sin \theta \\
-\cos \theta
\end{array}\right]+\frac{r t}{a}\left[\begin{array}{c}
\cos \theta \\
\sin \theta
\end{array}\right],} \\
{\left[\begin{array}{l}
x \\
y
\end{array}\right]=\left[\begin{array}{l}
x_{0} \\
y_{0}
\end{array}\right]+\frac{r}{a}\left[\begin{array}{c}
\cos \theta \\
\sin \theta
\end{array}\right]+r t\left[\begin{array}{c}
-\sin \theta \\
\cos \theta
\end{array}\right],} \\
{\left[\begin{array}{l}
x \\
y
\end{array}\right]=\left[\begin{array}{l}
x_{0} \\
y_{0}
\end{array}\right]+\frac{r}{a}\left[\begin{array}{c}
-\cos \theta \\
-\sin \theta
\end{array}\right]+r t\left[\begin{array}{c}
-\sin \theta \\
\cos \theta
\end{array}\right] .}
\end{gathered}
$$

The first two equations describe the short sides and the latter two describe the long ones.

Using equation (B7) in equations (B8) to (B11), we respectively have eight possible solutions ( 2 for each side):

$$
\begin{array}{r}
t=\frac{-\left(y_{0}+r \cos \theta\right) \pm a\left(x_{0}-r \sin \theta\right)}{r / a \sin \theta \mp r \cos \theta}, \\
t=\frac{-\left(y_{0}-r \cos \theta\right) \pm a\left(x_{0}+r \sin \theta\right)}{r / a \sin \theta \mp r \cos \theta}, \\
t=\frac{-1 / a\left(y_{0}+r / a \sin \theta\right) \pm\left(x_{0}+r / a \cos \theta\right)}{r / a \cos \theta \mp r \sin \theta}, \\
t=\frac{-1 / a\left(y_{0}-r / a \sin \theta\right) \pm\left(x_{0}-r / a \cos \theta\right)}{r / a \cos \theta \mp r \sin \theta} .
\end{array}
$$

Each of these has to be tested to see if $-1 \leq t \leq 1$. If so, then upon substituting the values of $t$ determined in in equations ( $\mathrm{B} 12)$ through $(\overline{\mathrm{B} 15})$ into equations $(\mathrm{B} 8)$ through (B11), we have

$$
\begin{gathered}
x=\frac{\left(x_{0} \sin \theta-y_{0} \cos \theta-r\right)}{\sin \theta \mp a \cos \theta}, \\
x=\frac{\left(x_{0} \sin \theta-y_{0} \cos \theta+r\right)}{\sin \theta \mp a \cos \theta}, \\
x=\frac{\left(x_{0} \cos \theta+y_{0} \sin \theta+r / a\right)}{\cos \theta \pm a \sin \theta}, \\
x=\frac{\left(x_{0} \cos \theta+y_{0} \sin \theta-r / a\right)}{\cos \theta \pm a \sin \theta},
\end{gathered}
$$

where $y= \pm a x$ in all cases.

We are now able to write down how to determine the size of the largest rectangle that may fit in given it is 
centered at the origin with an angle $\phi$ and there is one other rectangle already present at $\left(x_{0}, y_{0}\right)$ oriented at an angle $\theta$.

1. Determine whether or not the new rectangle lies within the old one using equation (B3).

2. If it is not enclosed, rotate the coordinate system by an angle $\phi$ so the central rectangle sits square with the axes. All that needs to be done is to move the existing rectangle from $\left(x_{0}, y_{0}\right)$ to $\left(\cos \phi x_{0}+\right.$ $\left.\sin \phi y_{0},-\sin \phi x_{0}+\cos \phi y_{0}\right)$, and change its angle of rotation to $\theta^{\prime}=\theta-\phi \quad(\bmod \pi)$.

3. Find the position of the four corner points using equation (B4) with $\theta^{\prime}$ and $\left(x_{0}^{\prime}, y_{0}^{\prime}\right)$.

4. Determine which, if any, intersection points with the diagonals of the new rectangle exist by calculating $t$ in equations (B12) through (B15).

5. Each value of $t$ that satisfies $-1 \leq t \leq 1$, is a valid intersection point. The positions of all such points are then taken from equations (B16) through (B19) along with $y= \pm a x$.

6. For each valid $(x, y)$ pair, calculate $\max (|a x|,|y|)$ which gives us the half-width of the shortest side of the rectangle centered at the origin that passes through the point $(x, y)$. Take the minimum over all such values to find the half-width of the shortest side of the actual largest rectangle that may be inserted without overlapping.
[1] P. S. Dodds and J. S. Weitz, Phys. Rev. E 65, 056108 (2002).

[2] B. B. Mandelbrot, Fractals: Form, Chance, and Dimension (Freeman, San Francisco, 1977).

[3] N. V. Brilliantov, P. L. Krapivsky, and Y. A. Andrienkov, J. Phys. A: Math. Gen. 27, L381 (1994).

[4] Y. A. Andrienko, N. V. Brilliantov, and P. L. Krapivsky, J. Stat. Phys. 75(3-4), 507 (1994).

[5] T. Huillet, J. Math. Chem. 24(1-3), 187 (1998).
[6] J. Conway and N. Sloane, Sphere Packings, Lattices and Groups (Springer-Verlag, New York, 1999), 3rd ed.

[7] S. S. Manna, Physica A 187(3-4), 373 (1992).

[8] T. Aste, Phys. Rev. E 53, 2571 (1996).

[9] C. A. Voigt and R. M. Ziff, Phys. Rev. E 56, R6241 (1997).

[10] P. S. Dodds and D. H. Rothman, Phys. Rev. E 63(1), 016115 (2001). 